\title{
Induced gravity and entanglement entropy of 2D black holes
}

\author{
Mariano Cadoni* \\ Dipartimento di Fisica, Università di Cagliari \\ and I.N.F.N., Sezione di Cagliari, \\ Cittadella Universitaria, 09042 Monserrato (Italy) \\ E-mail: mariano.cadoni@ca.infn.it
}

\begin{abstract}
Using the fact that 2D Newton constant is wholly induced by a conformal field theory, we derive a formula for the entanglement entropy of the anti-de Sitter black hole in two spacetime dimensions. The leading term in the large black hole mass expansion of our formula reproduces exactly the Bekenstein-Hawking entropy $\mathrm{S}_{B H}$, whereas the subleading term behaves as $\ln \mathrm{S}_{B H}$. This subleading term has the universal form typical for the entanglement entropy of physical systems described by effective conformal fields theories (e.g. one-dimensional statistical models at the critical point).
\end{abstract}

From Quantum to Emergent Gravity: Theory and Phenomenology

June 11-15 2007

Trieste, Italy

\footnotetext{
${ }^{*}$ Speaker.
} 


\section{Introduction}

There have always been two opposite points of view about the origin of Bekenstein-Hawking $(\mathrm{BH})$ black hole entropy. On the one side we have the assumption of an underlying unitary theory of quantum gravity $(\mathrm{QG})$ and the usual statistical paradigm explaining the $\mathrm{BH}$ entropy in terms of a microstate gas. On the opposite side we do not have necessarily an underlying unitary theory and $\mathrm{BH}$ entropy has to be thought as a fundamental feature of QG. Entanglement entropy (EE) is a notion which could be very useful to settle down the controversy.

Quantum entanglement is a fundamental feature of quantum systems. It is related to the existence of correlations between parts of the system. Its investigation has become increasingly relevant in many research areas. It is an old conjecture, first proposed by 'tHooft, that black hole entropy may be explained in terms of the entanglement of the quantum state of matter fields in the black hole geometry $[1,2,3,4,5,6,7,8,9,10]$. Support to this conjecture comes from the fact that both the EE of matter fields and the BH entropy depend on the area of the boundary region. However, any attempt to explain the $\mathrm{BH}$ entropy as due to quantum entanglement runs into conceptual and technical difficulties.

The usual statistical interpretation of the BH entropy is conceptually very different from the EE, which measures the observer's lack of information about the quantum state of the system in a inaccessible region of spacetime. Moreover, the EE depends both on the number of species $n_{s}$ of the matter fields, whose entanglement should reproduce the BH entropy and on the value of the UV cutoff $\varepsilon$ arising owing to the presence of a sharp boundary between the accessible and inaccessible regions of the spacetime. Conversely, the BH entropy is meant to be universal, independent both from $n_{s}$ and $\varepsilon$. Some conceptual problems look somehow milder using Sakharov's induced gravity approach $[11,12,13]$, but the problem of the dependence on $n_{s}$ and $\varepsilon$ is still there.

Recently, there have been considerable advances in the understanding of the EE entropy in field theory and condensed matter system. Exact formulas have been derived for the EE entropy of 2D CFTs [14, 15]. It has been shown that EE plays a important role in condensed matter systems, where it helps to understand quantum phases of matter (e.g spin chains and quantum liquids)[16, 17, 18, 19, 20]. Entanglement entropy turned out to be an useful concept also for investigating general features of quantum field theory (QFT), in particular two-dimensional conformal field theory (CFT), the Anti-de Sitter/conformal field theory (AdS/CFT) correspondence and the existence of c-theorems [21, 22, 23, 24, 25, 26].

One is therefore tempted to use this new ideas and improved understanding of the EE to tackle the old problems related to the EE entropy of black holes. In this contribution, which is mainly based on Ref. [27], we will show that this is possible at least in the case of two-dimensional AdS black holes. We will derive an expression for the black hole EE that in the large black hole mass limit reproduces exactly the $\mathrm{BH}$ entropy. Moreover, we will show that the subleading term has the universal behavior typical for CFTs and in particular for critical phenomena. The reason of this success is related to the peculiarities of 2D AdS gravity, namely the existence of an AdS/CFT correspondence and the fact that 2D Newton constant can be considered as wholly induced by quantum fluctuations of the dual CFT. 


\section{Entanglement entropy for 2D CFTs}

Most of the progress in understanding the EE in QFT has been achieved in the case of 2D CFT. If only a spacelike slice $Q$ (of length $\Sigma$ ) of our 2D universe, endowed with an IR cutoff $\Lambda$, is accessible for measurement we have to trace over the degrees of freedom in the complementary region $P$ (of length $\Lambda-\Sigma$ ). The entanglement entropy originated by tracing over the unobservable degrees of freedom is given by the Von Neumann entropy $S_{\text {ent }}=-T r_{Q} \hat{\rho}_{Q} \ln \hat{\rho}_{Q}$, where the reduced density matrix $\hat{\rho}_{Q}=\operatorname{Tr}_{P} \hat{\rho}$ is obtained by tracing the density matrix $\hat{\rho}$ over states in the region $P$. The resulting EE for the ground state of the 2D CFT has been first calculated by Holzhey, Larsen and Wilckzek [14]. Owing to the contribution of localized excitations arbitrarily near to the boundary the entanglement entropy diverges. Introducing an ultraviolet cutoff $\varepsilon$, the regularized entanglement entropy turns out to be

$$
S_{\text {ent }}=\frac{c+\bar{c}}{6} \ln \left(\frac{\Lambda}{\varepsilon \pi} \sin \frac{\pi \Sigma}{\Lambda}\right),
$$

where $c$ and $\bar{c}$ are the central charges of the 2D CFT.

Characterizing features of the expression (2.1) for the EE are:

a) Subadditivity

b) Invariance under the transformation which exchanges the inside and outside regions

$$
\Sigma \rightarrow \Lambda-\Sigma
$$

c) $S_{\text {ent }}$ is not a monotonic function of $\Sigma$, but increases and reaches its maximum for $\Sigma=\Lambda / 2$ and then decreases as $\Sigma$ increases further.

\section{2D AdS black hole and the AdS/CFT correspondence}

2D AdS black holes are classical solutions of a 2D (dilaton) gravity theory with action $A=$ $\int d^{2} x \sqrt{g} \Phi\left(R+2 / L^{2}\right)$, where the length $L$ is related to cosmological constant of the AdS spacetime $\left(\lambda=1 / L^{2}\right)$ and $\Phi$ is the dilaton. In the Schwarzschild gauge the 2D AdS black hole solutions are [28],

$$
d s^{2}=-\frac{1}{L^{2}}\left(r^{2}-r_{h}^{2}\right) d t^{2}+L^{2}\left(r^{2}-r_{h}^{2}\right)^{-1} d r^{2}, \quad \Phi=\frac{r}{G L},
$$

where $G$ is the dimensionless $2 \mathrm{D}$ Newton constant and $r_{h}$ is the horizon radius.

The black hole mass, temperature and Bekenstein-Hawking entropy are given by [28]

$$
M=\frac{r_{h}^{2}}{2 G L^{3}}, \quad T=\frac{r_{h}}{2 \pi L^{2}}, \quad S_{B H}=\frac{2 \pi r_{h}}{G L} .
$$

Setting $r_{h}=0$ in Eq. (3.1) we have the AdS black hole ground state with zero mass, temperature and entropy. The AdS black hole (3.1) can be considered as the thermalization of the ground state solution [28].

The 2D black hole has a dual description in terms of a CFT with central charge [29, 30, 31, 32]

$$
c=\frac{12}{G} .
$$


The dual CFT can have both the form of a 2D [31, 32] or a 1D [29, 30] conformal field theory. This $\mathrm{AdS}_{2} / \mathrm{CFT}_{2}$ ( or $\mathrm{AdS}_{2} / \mathrm{CFT}_{1}$ ) correspondence has been used to give a microscopical meaning to the thermodynamical entropy of 2D AdS black holes. The BH entropy (3.2) has been reproduced by counting states in the dual CFT.

\section{Entanglement entropy of the 2D AdS black hole}

It has been observed that in two dimensions black hole entropy can be ascribed to quantum entanglement if 2D Newton constant is wholly induced by quantum fluctuations of matter $[4,33,12,13]$. But Eq. (3.3) (or more in general the $\mathrm{AdS}_{2} / \mathrm{CFT}_{2}$ correspondence) tells us that the 2D Newton constant is induced by quantum fluctuations of the dual CFT. It follows that in the semiclassical approximation the black hole EE should be identified with the entanglement entropy of the vacuum for the 2D CFT of central charge given by Eq. (3.3) in the black hole geometry (3.1). The black hole exterior and interior should be identified respectively with the observable region $Q$ (of length $\Sigma$ ) and unobservable region $P$ (length $\Lambda-\Sigma$ ) of the 2D universe described above, where the CFT degrees of freedom live.

However, two obstacles prevent a direct application of Eq. (2.1):

1) Eq. (2.1) holds for a $2 D$ flat spacetime, whereas we are dealing with a curved $2 D$ background.

2) The calculations leading to Eq. (2.1) are performed for a spacelike slice $Q$, whereas in the black hole case, owing to the coordinate singularities at $r=r_{h}$ (the horizon) and $r=\infty$ (the timelike asymptotic boundary of the AdS spacetime), there is no global notion of a spacelike coordinate.

Owing to these geometrical features, in the black hole case we cannot give a direct meaning to both the measures $\Sigma$ and $(\Lambda-\Sigma)$ of the subsystems $Q, P$ and invariance under the transformation (2.2) becomes meaningless.

The second difficulty can be circumvented using appropriate coordinate system and regularization procedure, the first using instead of Eq. (2.1) the formula derived by Fiola et al. [4], which gives the EE of the vacuum of matter fields in the case of a curved background ${ }^{1}$,

$$
S_{\text {ent }}=-\frac{c}{6}\left(\left.\rho\right|_{\text {boundary }}-\ln \frac{\varepsilon}{\Lambda}\right)
$$

where $c$ is the central charge (3.3) and $\exp (2 \rho)$ is the conformal factor of the metric in the coordinate system used to define the CFT vacuum. In this coordinate system the black hole metric (3.1) takes the form [28]

$$
d s^{2}=\frac{r_{h}^{2}}{L^{2}} \frac{\left(-d t^{2}+d \sigma^{2}\right)}{\sinh ^{2}\left(\frac{r_{h} \sigma}{L^{2}}\right)} .
$$

Notice that in Eq. (4.1) we have contributions from only one sector (e.g. right movers) of the CFT. In Ref. $[31,32]$ it has been shown that the 2D AdS black hole is dual to an open string with appropriate boundary conditions. These boundary conditions are such that only one sector of the $\mathrm{CFT}_{2}$ is present.

\footnotetext{
${ }^{1}$ Notice that we are using the formula of Ref. [4] with reversed sign. The AdS black hole has to be considered as the vacuum seen by the observer using the black hole coordinates. This observer sees the the CFT vacuum as filled with thermal radiation with negative flux [28].
} 


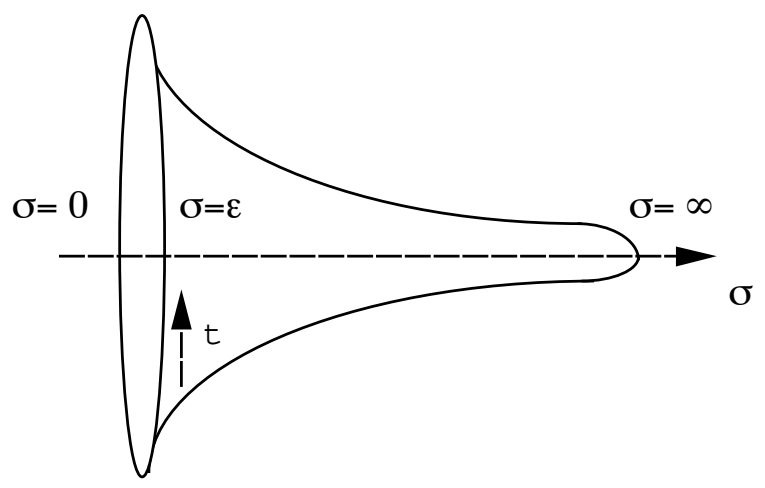

Figure 1: Regularized euclidean instanton corresponding to the 2D AdS black hole in the coordinate system $(t, \sigma)$ covering only the black hole exterior. The euclidean time is periodic. The point $\sigma=\infty$ correspond to the black hole horizon. $\sigma=0$ corresponds to the asymptotic timelike boundary of $\mathrm{AdS}_{2}$.

The coordinate system $(t, \sigma)$ covers only the black hole exterior. Working in euclidean space the 2D manifold has only a boundary at $\sigma=0$, corresponding to $r=\infty$, the timelike conformal boundary of the 2D AdS space. The conformal factor of the metric (4.2), hence the entanglement entropy (4.1), blows up on the $\sigma=0$ boundary of the AdS spacetime. The simplest regularization procedure is to consider a regularized boundary at $\sigma=\varepsilon$. Notice that $\varepsilon$ plays the role of a UV cutoff for the coordinate $\sigma$, which is the natural spacelike coordinate of the dual CFT. $\varepsilon$ is an IR cutoff for the coordinate $r$, which is the natural spacelike coordinate for the $\mathrm{AdS}_{2}$ black hole. The regularized euclidean instanton corresponding to the black hole (4.2) is shown in figure (1).

The regularized boundary is at finite proper distance from the horizon. $\varepsilon$ acts also as IR regulator, making the presence of the IR cutoff $\Lambda$ in Eq. (4.1) redundant. The EE still depends on the UV cutoff $\varepsilon$. The AdS/CFT correspondence enable us to identify $\varepsilon$ as the UV cutoff of the CFT: $\varepsilon \propto L$. The proportionality factor can be determined by requiring that the analytical continuation of Eq. (4.3) below is invariant under the transformation (2.2) (see later). This requirement fixes $\varepsilon=\pi L$.

Putting all together we obtain from Eq. (4.1) the EE entropy of the 2D CFT in the classical black hole geometry,

$$
S_{\text {ent }}^{(b h)}=\frac{c}{6} \ln \left(\frac{L}{\pi r_{h}} \sinh \frac{\pi r_{h}}{L}\right),
$$

which has to be identified with the EE of the 2D AdS black hole. The entanglement entropy (4.3) has the expected behavior as a function of the horizon radius $r_{h}$ or, equivalently, of the black 
hole mass $M . S_{\text {ent }}^{(b h)}$ becomes zero in the AdS ground state, $r_{h}=0(M=0)$, whereas it grows monotonically for $r_{h}>0(M>0)$.

Let us now consider the large mass behavior $r_{h}>>L$ (macroscopic black holes) of Eq. (4.3):

$$
S_{\text {ent }}^{(b h)}=\frac{2 \pi r_{h}}{G L}-\frac{2}{G} \ln \frac{r_{h}}{L}+O(1)=S_{B H}-\frac{2}{G} \ln S_{B H}+O(1) .
$$

We have obtained the remarkable result that the leading term in the large mass expansion of the black hole entanglement entropy reproduces exactly the Bekenstein-Hawking entropy. In this regime the thermal length $\beta=1 / T<<L$, thermal correlations dominate and the EE becomes Gibbs entropy. The subleading term has the universally predicted $\ln S_{B H}$ behavior for the quantum corrections to the Bekenstein-Hawking result. Notice that, although it is an universally accepted result that the quantum corrections to the BH entropy behave as $\ln S_{B H}[34,35,36,37,38,39,40$, $41,42,43]$, there is no general agreement about the value of the prefactor of this term. Equation (4.4) fixes the prefactor of $\ln S_{B H}$ in terms of the 2D Newton constant. This result contradicts some previous results supporting a $G$-independent value of the prefactor. Our result is consistent with the approach followed in this paper, which considers $2 \mathrm{D}$ gravity as induced from the quantum fluctuations of a CFT with central charge $12 / G$. The subleading term in Eq. (4.4) has also the universal behavior shared with other systems described by 2D QFTs, such as one-dimensional statistical models near to the critical point (with the black hole radius $r_{h}$ corresponding to the correlation length) or free scalars fields [15, 22].

For $\beta>>L$ quantum correlations dominate but we expect our semiclassical description, which also neglects back-reaction, to break down at $\beta \sim L$. In this regime it is likely that a phase transition, analogue to the Hawking-Page transition in $4 \mathrm{D}$ takes place.

The black hole EE (4.3) differs from the CFT formula (2.1) by the exchange of hyperbolic with trigonometric functions. The appearance of hyperbolic functions solve the problems concerning the application of formula (2.1) to the black hole case. It allows for monotonic increasing of $S_{e n t}^{(b h)}\left(r_{h}\right)$, eliminating the unphysical decreasing behavior of $S_{\text {ent }}(\Sigma)$ in the region $\Sigma>\Lambda / 2$.

On the other hand the presence of hyperbolic instead of trigonometric function indicates that Eq. (2.1) can be obtained as the analytic continuation $r_{h} \rightarrow i r_{h}$ of our formula (4.3), i.e by considering the solution (3.1) with negative mass. The analytically continued solution is given by Eq. (3.1) with $r_{h}{ }^{2}<0$. In the conformal gauge the solution reads now $d s^{2}=\left[r_{h} /\left(L \sin \left(r_{h} \sigma / L^{2}\right)\right]^{2}\left(-d t^{2}+\right.\right.$ $d \sigma^{2}$ ). The corresponding regularized euclidean instanton is shown in Fig. (2). In terms of the $2 \mathrm{D}$ CFT we have to trace over the degrees of freedom outside the spacelike slice $\varepsilon<\sigma<\pi L^{2} / 2 r_{h}$. Applying Eq. (4.1 to the case of a spacelike slice with two boundary points and redefining appropriately the UV cutoff $\varepsilon$, we get

$$
S_{\text {ent }}=\frac{c}{6} \ln \left(\frac{\Lambda}{\pi \varepsilon} \sin \frac{\pi r_{h}}{\Lambda}\right) .
$$

Thus, the entanglement entropy of the 2D CFT in the curved background given by the AdS solution with negative mass has exactly the form given by Eq. (2.1) with the horizon radius $r_{h}$ playing the role of $\Sigma$. The requirement that equation (4.5) is the analytic continuation of Eq. (4.3) fixes, as previously anticipated, the proportionality factor between $\varepsilon$ and $L$ in the calculations leading to Eq. (4.3). 


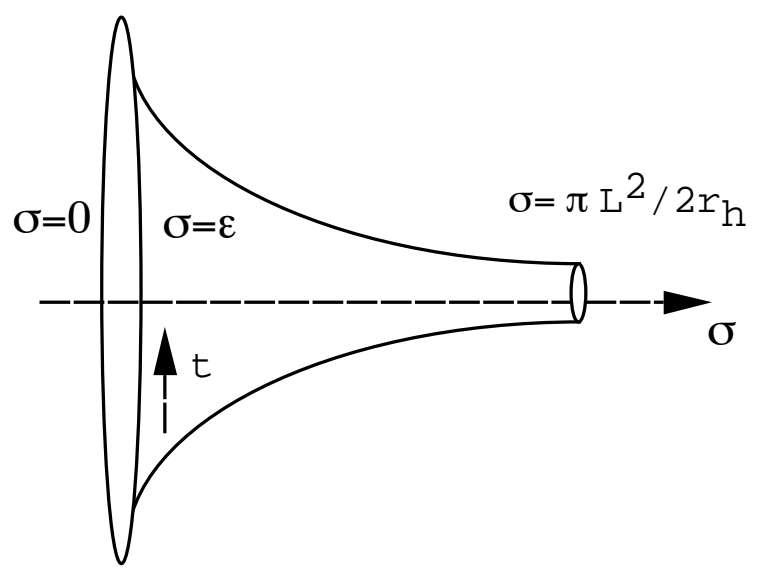

Figure 2: Regularized euclidean instanton corresponding to the 2D AdS solution with negative mass. The euclidean time is periodic. The point $\sigma=\pi L^{2} / 2 r_{h}$ corresponds to the black hole singularity at $r=0 . \sigma=0$ corresponds to the asymptotic timelike boundary of $\mathrm{AdS}_{2}$.

\section{Conclusions}

Thanks to the AdS/CFT correspondence we were able to solve the number of species and UV cutoff problem and to derive a formula for the entanglement entropy of 2D AdS black holes that has nice striking features. The leading term in the large black hole mass expansion reproduces exactly the BH entropy. The subleading term has the right $\ln S_{B H}$, behavior of the quantum corrections to the $\mathrm{BH}$ formula and represents an universal term typical of CFTs.

The picture that emerges is intriguing but to a large extend expected: the black hole EE entropy reduces to the Bekenstein-Hawking entropy only for macroscopic black holes when thermal correlations dominate. Away from this regime the EE codifies information about the full quantum gravity description. Black hole EE could be therefore used to gain information about quantum gravity phase transition (e.g Hawking-Page-like phase transition). In particular, it could be used to describe the emergence of a spacetime description from the microscopic theory, in the same way as it is used to gain information about quantum phase transitions in condensed matter systems. Obviously, in the full quantum gravity regime $\beta>>$ our description breaks down and the full underlying microscopic theory ( e.g string theory on $\mathrm{AdS}_{2}$ times some compact manifold) has to be used.

Generalization of our approach to higher dimensional gravity theory would be more then wel- 
comed. Our results rely heavily on peculiarities of 2D AdS gravity, namely the existence of an AdS/CFT correspondence and on the fact that 2D Newton constant arises from quantum fluctuation of the dual CFT. The generalization of our approach to higher dimensional gravity theories is therefore far from being trivial.

\section{References}

[1] G. 't Hooft, Nucl. Phys. B 256 (1985) 727.

[2] L. Bombelli, R. K. Koul, J. H. Lee and R. D. Sorkin, Phys. Rev. D 34 (1986) 373.

[3] V. P. Frolov and I. Novikov, Phys. Rev. D 48 (1993) 4545 [arXiv:gr-qc/9309001].

[4] T. M. Fiola, J. Preskill, A. Strominger and S. P. Trivedi, Phys. Rev. D 50 (1994) 3987 [arXiv:hep-th/9403137].

[5] F. Belgiorno and S. Liberati, Phys. Rev. D 53 (1996) 3172 [arXiv:gr-qc/9503022].

[6] S. Hawking, J. M. Maldacena and A. Strominger, JHEP 0105 (2001) 001 [arXiv:hep-th/0002145].

[7] J. M. Maldacena, JHEP 0304 (2003) 021 [arXiv:hep-th/0106112].

[8] R. Brustein, M. B. Einhorn and A. Yarom, JHEP 0601 (2006) 098 [arXiv:hep-th/0508217].

[9] R. Emparan, JHEP 0606 (2006) 012 [arXiv:hep-th/0603081].

[10] P. Valtancoli, arXiv:hep-th/0612049.

[11] T. Jacobson, arXiv:gr-qc/9404039.

[12] V. P. Frolov, D. V. Fursaev and A. I. Zelnikov, Nucl. Phys. B 486 (1997) 339 [arXiv:hep-th/9607104].

[13] V. P. Frolov and D. V. Fursaev, Phys. Rev. D 56 (1997) 2212 [arXiv:hep-th/9703178].

[14] C. Holzhey, F. Larsen and F. Wilczek, Nucl. Phys. B 424 (1994) 443 [arXiv:hep-th/9403108].

[15] P. Calabrese and J. L. Cardy, J. Stat. Mech. 0406 (2004) P002 [arXiv:hep-th/0405152].

[16] G. Vidal, J. I. Latorre, E. Rico and A. Kitaev, Phys. Rev. Lett. 90 (2003) 227902 [arXiv:quant-ph/0211074].

[17] A. R. Its, B. Q. Jin, V. E. Korepin, J. Phys. A 38 (2005) 2975 [arXiv:quant-ph/0409027].

[18] A. Kitaev and J. Preskill, Phys. Rev. Lett. 96 (2006) 110404 [arXiv:hep-th/0510092].

[19] J. I. Latorre, C. A. Lutken, E. Rico and G. Vidal, Phys. Rev. A 71 (2005) 034301 [arXiv:quant-ph/0404120].

[20] V. E. Korepin, Phys. Rev. Lett. 92 (2003) 964021.

[21] H. Casini and M. Huerta, Phys. Lett. B 600 (2004) 142 [arXiv:hep-th/0405111].

[22] D. V. Fursaev, Phys. Rev. D 73 (2006) 124025 [arXiv:hep-th/0602134].

[23] S. N. Solodukhin, Phys. Rev. Lett. 97 (2006) 201601 [arXiv:hep-th/0606205].

[24] S. Ryu and T. Takayanagi, Phys. Rev. Lett. 96 (2006) 181602 [arXiv:hep-th/0603001].

[25] S. Ryu and T. Takayanagi, JHEP 0608 (2006) 045 [arXiv:hep-th/0605073].

[26] V. E. Hubeny, M. Rangamani and T. Takayanagi, JHEP 0707 (2007) 062 [arXiv:0705.0016 [hep-th]]. 
[27] M. Cadoni, arXiv:0704.0140 [hep-th].

[28] M. Cadoni and S. Mignemi, Phys. Rev. D 51 (1995) 4319 [arXiv:hep-th/9410041].

[29] M. Cadoni and S. Mignemi, Phys. Rev. D 59 (1999) 081501 [arXiv:hep-th/9810251].

[30] M. Cadoni and S. Mignemi, Nucl. Phys. B 557 (1999) 165 [arXiv:hep-th/9902040].

[31] M. Cadoni and M. Cavaglia, Phys. Lett. B 499 (2001) 315 [arXiv:hep-th/0005179].

[32] M. Cadoni and M. Cavaglia, Phys. Rev. D 63 (2001) 084024 [arXiv:hep-th/0008084].

[33] L. Susskind and J. Uglum, Phys. Rev. D 50 (1994) 2700 [arXiv:hep-th/9401070].

[34] D. V. Fursaev, Phys. Rev. D 51 (1995) 5352 [arXiv:hep-th/9412161].

[35] R. B. Mann and S. N. Solodukhin, Nucl. Phys. B 523 (1998) 293 [arXiv:hep-th/9709064].

[36] R. K. Kaul and P. Majumdar, Phys. Rev. Lett. 84 (2000) 5255 [arXiv:gr-qc/0002040].

[37] S. Carlip, Class. Quant. Grav. 17 (2000) 4175 [arXiv:gr-qc/0005017].

[38] A. Ghosh and P. Mitra, Phys. Rev. Lett. 73 (1994) 2521 [arXiv:hep-th/9406210].

[39] S. Mukherji and S. S. Pal, JHEP 0205 (2002) 026 [arXiv:hep-th/0205164].

[40] M. R. Setare, Phys. Lett. B 573 (2003) 173 [arXiv:hep-th/0311106].

[41] M. Domagala and J. Lewandowski, Class. Quant. Grav. 21 (2004) 5233 [arXiv:gr-qc/0407051].

[42] A. J. M. Medved, Class. Quant. Grav. 22 (2005) 133 [arXiv:gr-qc/0406044].

[43] D. Grumiller, arXiv:hep-th/0506175. 